\title{
Short-term electricity prices forecasting in a competitive market by a hybrid PSO-ANFIS approach
}

\author{
H.M.I. Pousinho ${ }^{\mathrm{a}, \mathrm{b}}$, V.M.F. Mendes ${ }^{\mathrm{c}}$, J.P.S. Catalão ${ }^{\mathrm{a}, \mathrm{b}, *}$ \\ ${ }^{a}$ Department of Electromechanical Engineering, University of Beira Interior, R. Fonte do Lameiro, 6201-001 Covilha, Portugal \\ ${ }^{\mathrm{b}}$ Center for Innovation in Electrical and Energy Engineering, Instituto Superior Técnico, Technical University of Lisbon, Av. \\ Rovisco Pais, 1049-001 Lisbon, Portugal \\ ${ }^{\mathrm{c}}$ Departmental Area of Electrical Engineering and Automation, Instituto Superior de Engenharia de Lisboa, R. Conselheiro Emídio \\ Navarro, 1950-062 Lisbon, Portugal
}

Received 30 October 2009; received in revised form 23 December 2011

\begin{abstract}
In this paper, a novel hybrid approach is proposed for electricity prices forecasting in a competitive market, considering a time horizon of one week. The proposed approach is based on the combination of particle swarm optimization and adaptive-network based fuzzy inference system. Results from a case study based on the electricity market of mainland Spain are presented. A thorough comparison is carried out, taking into account the results of previous publications, to demonstrate its effectiveness regarding forecasting accuracy and computation time. Finally, conclusions are duly drawn.

(C) 2011 Elsevier Ltd. All rights reserved.
\end{abstract}

Keywords: Electricity market; Price forecasting; Swarm optimization; Neural networks; Fuzzy logic

\section{Introduction}

All over the world, the electricity industry is converging toward a competitive framework and a market environment is replacing the traditional monopolistic scenery for the electricity industry [1]. Deregulation brings electricity prices uncertainty, placing higher requirements on forecasting.

In most competitive electricity markets the series of prices presents the following features: high frequency, non-constant mean and variance, daily and weekly seasonality, calendar effect on weekend and public holidays, high volatility and high percentage of unusual prices [2]. Hence, price forecasting [3-5] is extremely important for all market players [6,7]. An accurate forecast of electricity prices is a very important tool for a generating company to develop an appropriate bidding strategy in the market and to optimally schedule its hydro $[8]$ and/or thermal $[9,10]$ resources.

* Corresponding author at: Department of Electromechanical Engineering, University of Beira Interior, R. Fonte do Lameiro, 6201-001 Covilha, Portugal. Tel.: +351275 329914; fax: +351275329972.

E-mail address: catalao@ubi.pt (J.P.S. Catalão). 
Several techniques have been tried out for electricity prices forecasting. In general, hard and soft computing [11] techniques could be used to forecast electricity prices.

The hard computing techniques include transfer function model [12], auto regressive integrated moving average (ARIMA) [13], wavelet-ARIMA [14], and mixed model [15] approaches. Usually, an exact model of the system is required, and the solution is found using algorithms that consider the physical phenomena that govern the process. Although these approaches can be very accurate, they require a lot of information, and the computational cost is very high.

The soft computing techniques include neural networks (NN) [16], neural networks combined with wavelet transform (NNWT) [17], fuzzy neural networks (FNN) [18], weighted nearest neighbors (WNN) [19], adaptive wavelet neural network (AWNN) [20], hybrid intelligent system (HIS) [21], cascaded neuro-evolutionary algorithm (CNEA) [22], and other hybrid approaches [23,24]. Usually, an inputoutput mapping is learned from historical examples, thus there is no need to model the system. Hence, these approaches can be much more efficient computationally and as accurate as the first ones, if the correct inputs are considered [25].

In this paper, a novel hybrid approach is proposed for short-term (one week) electricity prices forecasting. The proposed approach is based on the combination of particle swarm optimization (PSO) and adaptive-network based fuzzy inference system (ANFIS). Our HPA (hybrid PSO-ANFIS) approach is examined on the electricity market of mainland Spain, commonly used as the test case in several price forecasting papers [13-22]. It has been concluded that the Spanish market has a hard nonlinear behaviour and time variant functional relationship $[14,18]$. So, this market is a real world case study with sufficient complexity. The proposed approach is compared with ARIMA, mixed-model, NN, wavelet-ARIMA, WNN, FNN, HIS, AWNN, NNWT, and CNEA approaches, to demonstrate its effectiveness regarding forecasting accuracy and computation time.

The paper is structured as follows. Section 2 presents the proposed approach to forecast electricity prices. Section 3 provides the different criterions used to evaluate the forecasting accuracy. Section 4 provides the results from a case study based on the electricity market of mainland Spain. Section 5 provides conclusions. 


\section{Proposed approach}

The proposed approach is based on a combination of PSO and ANFIS. The PSO is used to improve the performance of ANFIS, tuning the membership functions required to achieve a lower error.

\subsection{Particle Swarm Optimization}

Particle swarm optimization is a heuristic approach first proposed by Kennedy and Eberhart in 1995 [26] as an evolutionary computational method developed for dealing with the optimization of continuous and discontinuous function decision making. The PSO algorithm is based on the biological and sociological behavior of animals such as schools of fish and flocks of birds searching for their food [27].

PSO is a population-based search method where each potential solution is represented as a particle in a population (called swarm). Particles change their position in a multidimensional search space until equilibrium or optimal state has been reached or until computation limitations are exceeded. Empirical evidence has been accumulated to show that the algorithm is a useful tool for optimization [28].

Consider an optimization problem of $D$ variables. A swarm of $N$ particles is initialized in which each particle is assigned a random position in the $D$-dimensional hyperspace such that each particle's position corresponds to a candidate solution for the optimization problem. Let $x$ denote a particle's position (coordinate) and $v$ denote the particle's flight velocity over a solution space. Each individual $x$ in the swarm is scored using a scoring function that obtains a fitness value representing how good it solves the problem.

The best previous position of a particle is Pbest. The index of the best particle among all particles in the swarm is Gbest. Each particle records its own personal best position (Pbest), and knows the best positions found by all particles in the swarm (Gbest). Then, all particles that fly over the $D$-dimensional solution space are subject to updated rules for new positions, until the global optimal position is found. Velocity and position of a particle are updated by the following stochastic and deterministic update rules:

$v_{i}(t)=\omega v_{i}(t-1)+\rho_{1}\left(x_{\text {Pbest }_{i}}-x_{i}(t)\right)+\rho_{2}\left(x_{\text {Gbest }}-x_{i}(t)\right)$

$x_{i}(t)=x_{i}(t-1)+v_{i}(t)$

where $\omega$ is an inertia weight, $\rho_{1}$ and $\rho_{2}$ are random variables. The random variables are defined as $\rho_{1}=r_{1} c_{1}$ and $\rho_{2}=r_{2} c_{2}$, with $r_{1}, r_{2} \sim U(0,1)$, and $C_{1}$ and $C_{2}$ are positive acceleration constants. 
Fig. 1 illustrates a search mechanism of a PSO technique using the velocity update rule (1) and the position update rule (2).

"See Fig. 1 at the end of the manuscript".

Acceleration constants $C_{1}$ and $C_{1}$ represent the weights of the stochastic acceleration terms that push a particle toward Pbest and Gbest, respectively. Small values allow a particle to roam far from target regions. Conversely, large values result in the abrupt movement of particles toward target regions. In this work, constants $C_{1}$ and $C_{1}$ are both set at 2.0, following the typical practice in [29]. Suitable correction of inertia $\omega$ in (2) provides a balance between global and local explorations, thereby reducing the number of iterations when finding a sufficiently optimal solution. An inertia correction function called "inertia weight approach (IWA)" is utilized in this work [29]. During the IWA, the inertia weight $\omega$ is modified according to the following equation:

$\omega=\omega_{\max }-\frac{\omega_{\max }-\omega_{\min }}{I t r_{\max }} I t r$

where $\omega_{\max }$ and $\omega_{\min }$ are the initial and final inertia weights, $I t r_{\max }$ is the maximum number of iteration, and Itr is the current number of iteration.

2.2 ANFIS

$\mathrm{NN}$ are simple, but powerful and flexible tools for forecasting, provided that there are enough data for training, an adequate selection of the input-output samples, an appropriated number of hidden units and enough computational resources available. Also, NN have the well-known advantages of being able to approximate any nonlinear function and being able to solve problems where the input-output relationship is neither well defined nor easily computable, because NN are data-driven. Multi-layered feedforward NN are specially suited for forecasting, implementing nonlinearities using sigmoid functions for the hidden layer and linear functions for the output layer [16].

Just like NN, a fuzzy logic system is a nonlinear mapping of an input vector into a scalar output, but it can handle numerical values and linguistic knowledge. In general, a fuzzy logic system contains four components: fuzzifier, rules, inference engine, and defuzzifier. The fuzzifier converts a crisp input variable into a fuzzy representation, where membership functions give the degree of belonging of the variable to a given attribute. Fuzzy rules are of the type "if-then", and can be derived from numerical data or from expert linguistic. Mamdani and Sugeno inference engines are two of the main types of inference mechanisms $[30,31]$. 
The Mamdani engine combines fuzzy rules into a mapping from fuzzy input sets to fuzzy output sets, while the Takagi-Sugeno type relates fuzzy inputs and crisp outputs. The defuzzifier converts a fuzzy set into a crisp number using the centroid of area, bisector of area, mean of maxima, or maximum criteria $[30,31]$.

NN have the advantage over the fuzzy logic models that knowledge is automatically acquired during the learning process. However, this knowledge cannot be extracted from the trained network behaving as a black box. Fuzzy systems, on the other hand, can be understood through their rules, but these rules are difficult to define when the system has too many variables and their relations are complex [25].

A combination of NN and fuzzy systems has the advantages of each of them. In a neuro-fuzzy system, neural networks extract automatically fuzzy rules from numerical data and, through the learning process, the membership functions are adaptively adjusted.

ANFIS is a class of adaptive multi-layer feedforward networks, applied to nonlinear forecasting where past samples are used to forecast the sample ahead. ANFIS incorporates the self-learning ability of NN with the linguistic expression function of fuzzy inference [32].

The ANFIS architecture is shown in Fig. 2. The ANFIS network is composed of five layers. Each layer contains several nodes described by the node function. The node function is described next. Let $O_{i}^{j}$ denote the output of the $i$ th node in layer $j$.

"See Fig. 2 at the end of the manuscript".

In layer 1, every node $i$ is an adaptive node with node function:

$O_{i}^{1}=\mu A_{i}(x), \quad i=1,2$

or

$O_{i}^{1}=\mu B_{i-2}(y), \quad i=3,4$

where $x$ (or $y$ ) is the input to the $i$ th node and $A_{i}\left(\right.$ or $\left.B_{i-2}\right)$ is a linguistic label associated with this node.

Thus, $O_{i}^{1}$ is the membership grade of a fuzzy set $A\left(=A_{1}, A_{2}, B_{1}\right.$, or $\left.B_{2}\right)$ and it specifies the degree to which the given input $x$ (or $y$ ) satisfies the quantifier $A$. The membership functions for $A$ and $B$ are usually described by generalized bell functions, e.g.: 
$\mu A_{i}(x)=\frac{1}{1+\left|\frac{x-r_{i}}{p_{i}}\right|^{2 q_{i}}}$

where $\left\{p_{i}, q_{i}, r_{i}\right\}$ is the parameter set. As the values of these parameters change, the bell-shaped function varies accordingly, thus exhibiting various forms of membership functions on linguistic label $A_{i}$.

In fact, any continuous and piecewise differentiable functions, such as triangular-shaped membership functions, are also qualified candidates for node functions in this layer. Parameters in this layer are referred to as premise parameters.

In layer 2, each node $\prod$ multiplies incoming signals and sends the product out:

$O_{i}^{2}=w_{i}=\mu A_{i}(x) \mu B_{i}(y), \quad i=1,2$

Hence, each node output represents the firing strength of a rule.

In layer 3, each node $\mathrm{N}$ computes the ratio of the ith rules's firing strength to the sum of all rules' firing strengths:

$O_{i}^{3}=\bar{w}_{i}=\frac{w_{i}}{w_{1}+w_{2}}, \quad i=1,2$

The outputs of this layer are called normalized firing strengths.

In layer 4, each node computes the contribution of the ith rule to the overall output:

$O_{i}^{4}=\bar{w}_{i} z_{i}=\bar{w}_{i}\left(a_{i} x+b_{i} y+c_{i}\right), \quad i=1,2$

where $\bar{w}_{i}$ is the output of layer 3 and $\left\{a_{i}, b_{i}, c_{i}\right\}$ is the parameter set. Parameters of this layer are referred to as consequent parameters.

In layer 5, the single node $\sum$ computes the final output as the summation of all incoming signals:

$$
O_{i}^{5}=\sum_{i} \bar{w}_{i} z_{i}=\frac{\sum_{i} w_{i} z_{i}}{\sum_{i} w_{i}}
$$

Thus, an adaptive network is functionally equivalent to a Sugeno-type fuzzy inference system. 
In this paper, ANFIS employs PSO method to adjust the parameters of the membership functions, as in [33]. The PSO techniques have the advantage of being less computationally expensive for a given size of network topology. The membership functions considered in this study are triangular-shaped.

\subsection{Hybrid Approach}

In this section, the algorithm used to implement the proposed approach is described step-by-step and presented in Fig. 3.

"See Fig. 3 at the end of the manuscript".

1) First step: Form a matrix with a set of historical data on electricity prices, arranged in $C$ columns of a matrix thereof. Each column of the array has an associated profile of prices for a particular week where prices are known beforehand. In this first step the matrix has 6 columns, corresponding to the 6 previous weeks to the week whose prices are to be forecasted.

2) Second step: Select a number of columns of the previous array so that the set of values derived from it represents the real input data. In this step, appropriate inputs are selected based on a correlation analysis. The candidate inputs with correlation coefficient greater than 0.8 are selected, corresponding to 4 of the 6 weeks with the highest correlation.

4) Fourth step: Get the signal so that it can be submitted to the entrance of the ANFIS structure.

5) Fifth step: Train the ANFIS with the data from the implementation of the previous step. The training process allows the system to adjust its parameters as inputs/outputs submitted. The training process stops whenever the designated number of times is reached or the objective of training error is achieved. The number of epochs used was eighteen. This was achieved by trial and error. After defining the training data, the number of times and the type of membership functions, the system of neuro-fuzzy inference is optimized by adapting the parameters of membership functions. The PSO is used to train the parameters associated with the membership functions of fuzzy inference system.

6) Sixth step: Create a vector with N-dimension, where $N$ equals the number of membership functions. This vector contains the parameters of membership function and will be further optimized by PSO algorithm. The fitness function is defined as the mean squared error.

7) Seventh step: Define the parameters associated with the PSO algorithm, provided in Table 1.

"See Table 1 at the end of the manuscript". 
Parameters are initialized randomly in first stage and then are being updated using PSO algorithm. In each iteration, one of the parameters of membership function is being updated. i.e. in the first iteration for example $p_{i}$ is updated then in second iteration $q_{i}$ is updated and then after updating all parameters again the first parameter update is considered and so on. These parameters are grouped in a vector that is being updated iteration to iteration. The PSO algorithm used to optimize parameters of membership functions is described below: (i) initialize the population positions and speeds. For each particle, the position and velocity vectors are randomly initialized with the same size as presented by the size of the problem; (ii) assess the ability of the individual particle (Pbest). If the value is better than the current value of the individual particle, Pbest reset the current position of the particle and update the individual value. If the best of all the particles of individual values is better than the overall value of current Gbest reset the location of the best particles; (iii) measure the fitness of each particle (Pbest) and store the particles with the best value of fitness (Gbest); (iv) modify the speed based on the position Pbest and Gbest; (v) update the particles; (vi) end if the condition is verified. If the current iteration number reaches the maximum default number or the result reached a minimum error set, then stop the iteration and collect the best solution.

8) Eighth step: Extract the output of the ANFIS using the parameters found by the PSO.

9) Ninth step: The final output corresponds to the prediction of our hybrid HPA approach.

\section{Forecasting accuracy evaluation}

To evaluate the accuracy of the HPA approach in forecasting electricity prices, different criterions are used. This accuracy is computed in function of the actual prices that occurred. The mean absolute percentage error (MAPE) criterion, the sum squared error (SSE) criterion, and the standard deviation of error (SDE) criterion, are defined as follows.

The MAPE criterion is defined as follows:

$$
\begin{aligned}
& \text { MAPE }=\frac{100}{N} \sum_{h=1}^{N} \frac{\left|\hat{p}_{h}-p_{h}\right|}{\bar{p}} \\
& \bar{p}=\frac{1}{N} \sum_{h=1}^{N} p_{h}
\end{aligned}
$$

where $\hat{p}_{h}$ and $p_{h}$ are respectively the forecasted and actual electricity prices at hour $h, \bar{p}$ is the average price of the forecasting period and $N$ is the number of forecasted hours. 
Electricity price can rise to tens or even hundreds of times of its normal value at particular hours, and it may drop to zero at other hours. Hence, the average price is used in (10) to avoid the adverse effect of prices close to zero [34].

The SSE criterion is given by:

$S S E=\sum_{h=1}^{N}\left(\hat{p}_{h}-p_{h}\right)^{2}$

The SDE criterion is given by:

$$
\begin{aligned}
& S D E=\sqrt{\frac{1}{N} \sum_{h=1}^{N}\left(e_{h}-\bar{e}\right)^{2}} \\
& e_{h}=\hat{p}_{h}-p_{h} \\
& \bar{e}=\frac{1}{N} \sum_{h=1}^{N} e_{h}
\end{aligned}
$$

where $e_{h}$ is the forecast error at hour $h$ and $\bar{e}$ is the average error of the forecasting period.

A measure of the uncertainty of a model is the variability of what is still unexplained after fitting the model, which can be measured through the estimation of the variance of the error. The smaller this variance, the more precise is the prediction of prices [14].

Consistent with definition (11), weekly error variance can be estimated as

$$
\begin{gathered}
\sigma_{e, \text { week }}^{2}=\frac{1}{N} \sum_{h=1}^{N}\left(\frac{\left|\hat{p}_{h}-p_{h}\right|}{\bar{p}}-\left(e_{\text {week }}\right)\right)^{2} \\
e_{\text {week }}=\frac{1}{N} \sum_{h=1}^{N} \frac{\left|\hat{p}_{h}-p_{h}\right|}{\bar{p}}
\end{gathered}
$$

The weekly MAPE and error variance are used in the case study.

\section{Numerical results}

The HPA approach has been applied to forecast next-week prices in the electricity market of mainland Spain. Price forecasting is computed using historical data of year 2002 for the Spanish market. 
For the sake of simplicity and clear comparison, no exogenous variables are considered. Also, for the sake of a fair comparison, the same test weeks as in [13-22] are selected, which correspond to the four seasons of year 2002. To build the forecasting model, the hourly historical price data of the 42 days previous to the day of the week whose prices are to be forecasted have been considered.

Numerical results with the HPA approach are shown in Figs 4-7 respectively for the winter, spring, summer and fall weeks. Each figure shows the actual prices, solid line, together with the forecasted prices, dash-dot line.

"See Fig. 4 at the end of the manuscript".

"See Fig. 5 at the end of the manuscript".

"See Fig. 6 at the end of the manuscript".

"See Fig. 7 at the end of the manuscript".

Table 2 presents the values for the criterions to evaluate the accuracy of the HPA approach in forecasting electricity prices. The first column indicates the week, the second column presents the MAPE in percent, the third column presents the square root of the SSE, and the fourth column presents the SDE.

"See Table 2 at the end of the manuscript".

Table 3 shows a comparison between the HPA approach and the other approaches (ARIMA, mixedmodel, NN, wavelet-ARIMA, WNN, FNN, HIS, AWNN, NNWT and CNEA), regarding the MAPE criterion.

"See Table 3 at the end of the manuscript".

The HPA approach presents enhanced forecasting accuracy, outperforming the other approaches. The MAPE for the Spanish market has an average value of 5.28\%.

In addition to the MAPE, stability of results is another important factor for the comparison of forecast approaches. Fig 8 and Table 4 show a comparison between the hybrid HPA approach and eight other approaches (ARIMA, NN, wavelet-ARIMA, FNN, HIS, AWNN, NNWT and CNEA), regarding weekly error variances.

"See Fig. 8 at the end of the manuscript".

"See Table 4 at the end of the manuscript".

The average error variance is smaller for the hybrid HPA approach, indicating less uncertainty in the predictions. 
Moreover, the average computation time is less than 5 seconds, using MATLAB on a PC with 1 GB of RAM and a 2.0-GHz-based processor. Instead, the computation time required by the proposed CNEA approach [22] is about 40 minutes. Hence, the proposed approach is both novel and effective for shortterm electricity prices forecasting.

\section{Conclusion}

A novel hybrid approach is proposed in this paper for short-term electricity prices forecasting, based on the combination of particle swarm optimization and adaptive-network-based fuzzy inference system. The application of the proposed approach to electricity prices forecasting on the Spanish market is both novel and effective. The effectiveness of this approach is thoroughly assessed by comparing it with techniques proposed by other authors. The HPA approach presents a good forecasting accuracy, with an average MAPE of $5.28 \%$ and weekly error variance of 0.0026 , taking into account the results of previous publications. Also, a reduced computation time is required, less than 5 seconds.

\section{Acknowledgements}

This work is funded by FEDER funds (European Union) through the Operational Programme for Competitiveness Factors - COMPETE, by Portuguese funds through the Fundação para a Ciência e a Tecnologia - FCT, under Project No. FCOMP-01-0124-FEDER-014887 (Ref. FCT PTDC/EEAEEL/110102/2009). Also, H.M.I. Pousinho thanks FCT for a Ph.D. Grant (SFRH/BD/62965/2009).

\section{References}

[1] Conejo AJ, Contreras J, Espínola R, Plazas MA. Forecasting electricity prices for a day-ahead pool-based electric energy market. Int J Forecasting 2005;21(3):435- 62.

[2] Nogales FJ, Contreras J, Conejo AJ, Espínola R. Forecasting next-day electricity prices by time series models. IEEE Trans Power Syst 2002;17(2):342-8.

[3] Unsihuay-Vila C, Zambroni de Souza AC, Marangon-Lima JW, Balestrassi PP. Electricity demand and spot price forecasting using evolutionary computation combined with chaotic nonlinear dynamic model. Electr Power Energy Syst 2010;32(2):108-16.

[4] Karsaz A, Mashhadi HR, Mirsalehi MM. Market clearing price and load forecasting using cooperative co-evolutionary approach. Electr Power Energy Syst 2010;32(5):408-15.

[5] Singhal D, Swarup KS. Electricity price forecasting using artificial neural networks. Electr Power Energy Syst 201 1;33(3):5505.

[6] Karandikar RG, Khaparde SA, Kulkarni SV. Strategic evaluation of bilateral contract for electricity retailer in restructured power market. Electr Power Energy Syst 2010;32(5):457-63.

[7] Das TK, Rocha P, Babayigit C. A matrix game model for analyzing FTR bidding strategies in deregulated electric power markets. Electr Power Energy Syst 2010;32(7):760-8. 
[8] Catalão JPS, Pousinho, HMI, Mendes VMF. Scheduling of head-dependent cascaded reservoirs considering discharge ramping constraints and start/stop of units. Electr Power Energy Syst 2010;32(8):904-10.

[9] Kazempour SJ, Moghaddam MP. Risk-constrained self-scheduling of a fuel and emission constrained power producer using rolling window procedure. Electr Power Energy Syst 2011;33(2):359-68.

[10] Catalão JPS, Mariano SJPS, Mendes VMF, Ferreira LAFM. A practical approach for profit-based unit commitment with emission limitations. Electr Power Energy Syst 2010;32(3):218-24.

[11] Szkuta BR, Sanabria LA, Dillon TS. Electricity price short-term forecasting using artificial neural networks. IEEE Trans Power Syst 1999;14(3):851-7.

[12] Nogales FJ, Conejo AJ. Electricity price forecasting through transfer function models. J Oper Res 2006;57(4):350-6.

[13] Contreras J, Espínola R, Nogales FJ, Conejo AJ. ARIMA models to predict next-day electricity prices. IEEE Trans Power Syst 2003;18(3):1014-20.

[14] Conejo AJ, Plazas MA, Espínola R, Molina AB. Day-ahead electricity price forecasting using the wavelet transform and ARIMA models. IEEE Trans Power Syst 2005;20(2):1035-42.

[15] Garcia-Martos C, Rodriguez J, Sanchez MJ. Mixed models for short-run forecasting of electricity prices: Application for the Spanish market. IEEE Trans Power Syst 2007;22(2):544-52.

[16] Catalão JPS, Mariano SJPS, Mendes VMF, Ferreira LAFM. Short-term electricity prices forecasting in a competitive market: a neural network approach. Electr Power Syst Res 2007;77(10):1297-304.

[17] Catalão JPS, Pousinho HMI, Mendes VMF. Neural networks and wavelet transform for short-term electricity prices forecasting. In: Proceedings of the 15th int. conf. on intelligent system applications to power systems. Curitiba, Brazil; 2009. [18] Amjady N. Day-ahead price forecasting of electricity markets by a new fuzzy neural network. IEEE Trans Power Syst 2006;21(2):887-96.

[19] Lora AT, Santos JMR, Expósito AG, Ramos JLM, Santos JCR. Electricity market price forecasting based on weighted nearest neighbors techniques. IEEE Trans Power Syst 2007;22(3):1294-301.

[20] Pindoriya NM, Singh SN, Singh SK. An adaptive wavelet neural network-based energy price forecasting in electricity markets. IEEE Trans Power Syst 2008;23(3):1423-32.

[21] Amjady N, Hemmati H. Day-ahead price forecasting of electricity markets by a hybrid intelligent system. Eur Trans Elect Power 2009;19(1):89-102.

[22] Amjady N, Keynia F. Day-Ahead price forecasting of electricity markets by mutual information technique and cascaded neuro evolutionary algorithm. IEEE Trans Power Syst 2009;24(1):306-18.

[23] Amjady N, Keynia F. Day ahead price forecasting of electricity markets by a mixed data model and hybrid forecast method. Electr Power Energy Syst 2008;30(9):533-46.

[24] Catalao JPS, Pousinho HMI, Mendes VMF. Hybrid Wavelet-PSO-ANFIS approach for short-term electricity prices forecasting. IEEE Trans Power Syst 2011;26(1):137-44.

[25] Rodriguez CP, Anders GJ. Energy price forecasting in the Ontario competitive power system market. IEEE Trans Power Syst 2004;19(1):366-74.

[26] Kennedy J, Eberhart R. Particle swarm optimization. In: Proceedings of the IEEE int. conf. neural networks; 1995.

[27] Bashir ZA, El-Hawary ME. Applying wavelets to short-term load forecasting using PSO-based neural networks. IEEE Trans Power Syst 2009;24(1):20-7.

[28] Yu W, Li X. Fuzzy identification using fuzzy neural networks with stable learning algorithms. IEEE Trans Fuzzy Syst 2004;12(3):411-20.

[29] Kennedy J. The behavior of particle. In Proceedings of the 7th annu. conf. evol. program; 1998.

[30] Shoorehdeli MA, Teshnehlab M, Sedigh AK, M. Khanesar A. Identification using ANFIS with intelligent hybrid stable learning algorithm approaches and stability analysis of training methods. Applied Soft Computing 2009;9(2):833-50.

[31] Jang JSR. ANFIS: adaptive-network-based fuzzy inference system. IEEE Trans Syst Man Cybern 1993;23(3):665-85.

[32] Yun Z, Quan Z, Caixin S, Shaolan L, Yuming L, Yang S. RBF neural network and ANFIS-based shortterm load forecasting approach in real-time price environment. IEEE Trans Power Syst 2008;23(3):853-8. 
[33] Shoorehdeli MA, Teshnehlab M, Sedigh AK, Khanesar MA. Identification using ANFIS with intelligent hybrid stable learning algorithm approaches and stability analysis of training methods. Appl Soft Comput 2009;9(2):833-50.

[34] Shahidehpour M, Yamin H, Li Z. Market operations in electric power systems: forecasting, scheduling and risk management. New York: Wiley; 2002. 
Figure captions

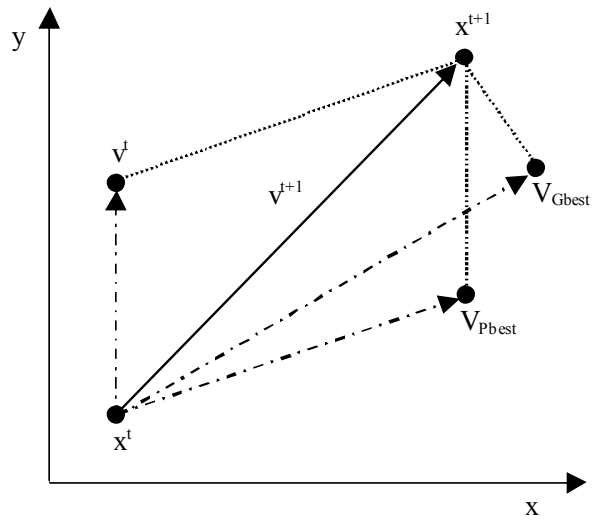

Fig. 1. Updating the position mechanism of PSO.

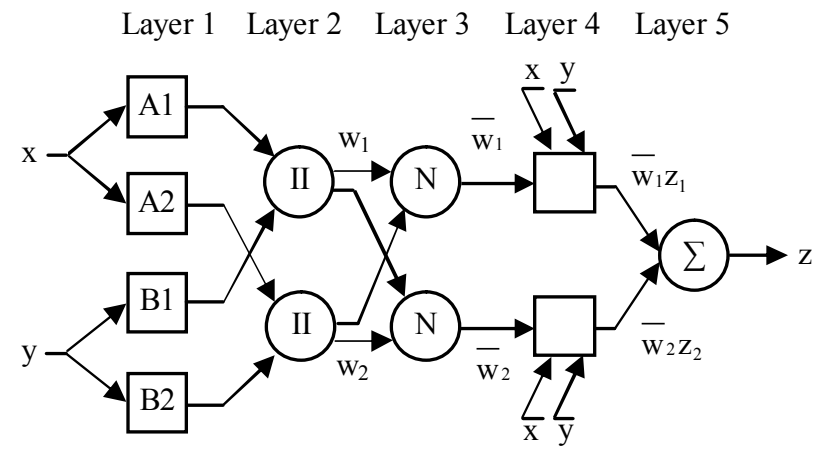

Fig. 2. ANFIS architecture. 


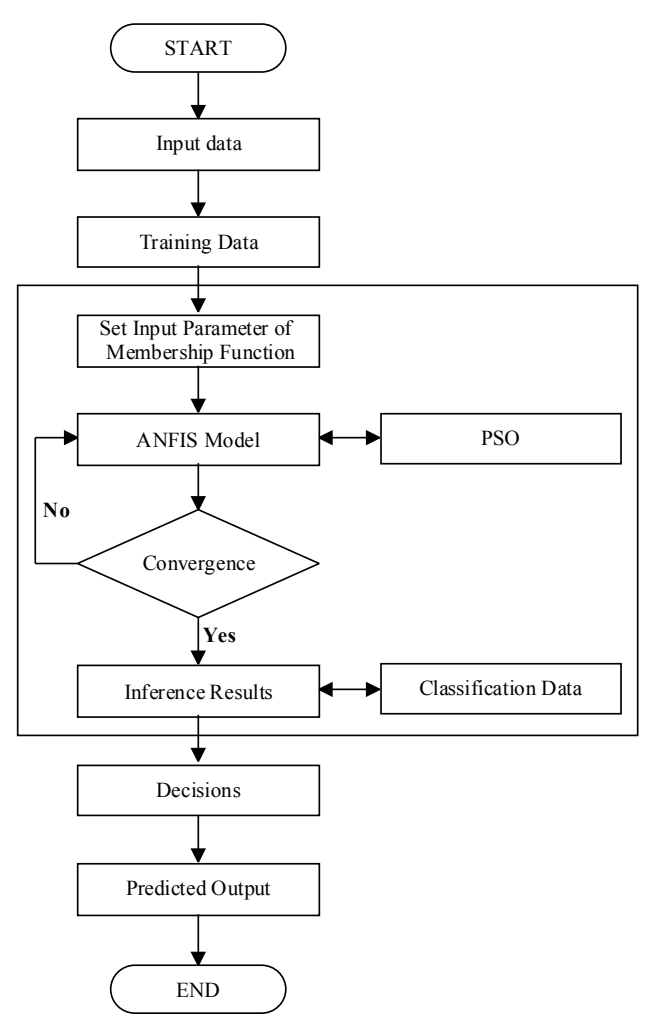

Fig. 3. Flowchart of the proposed hybrid approach.

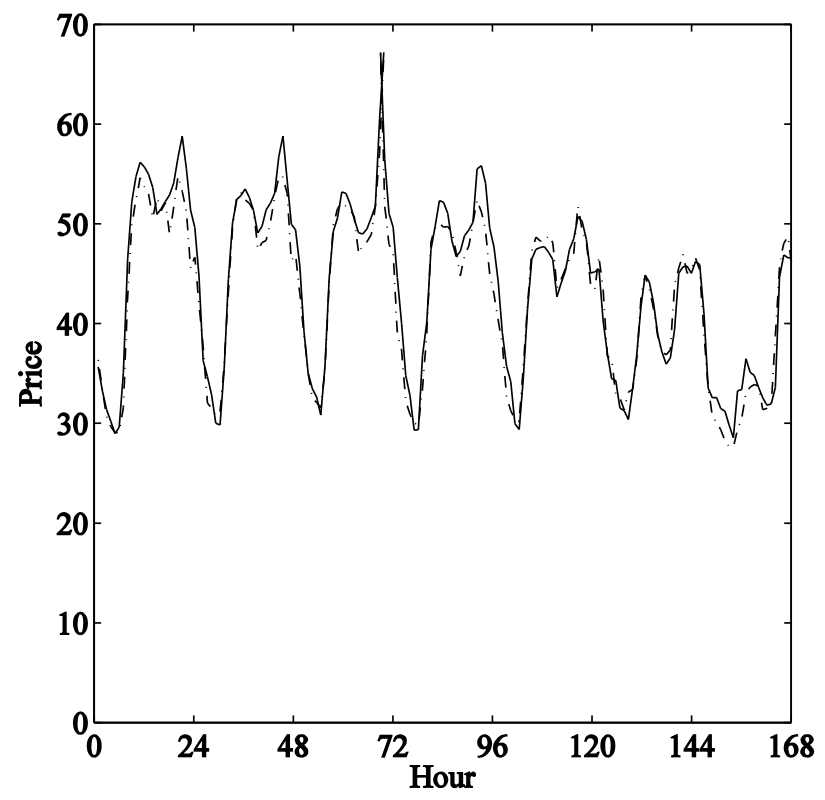

Fig. 4. Winter week: actual prices, solid line, together with the forecasted prices, dash-dot line, in euro per megawatt hour. 


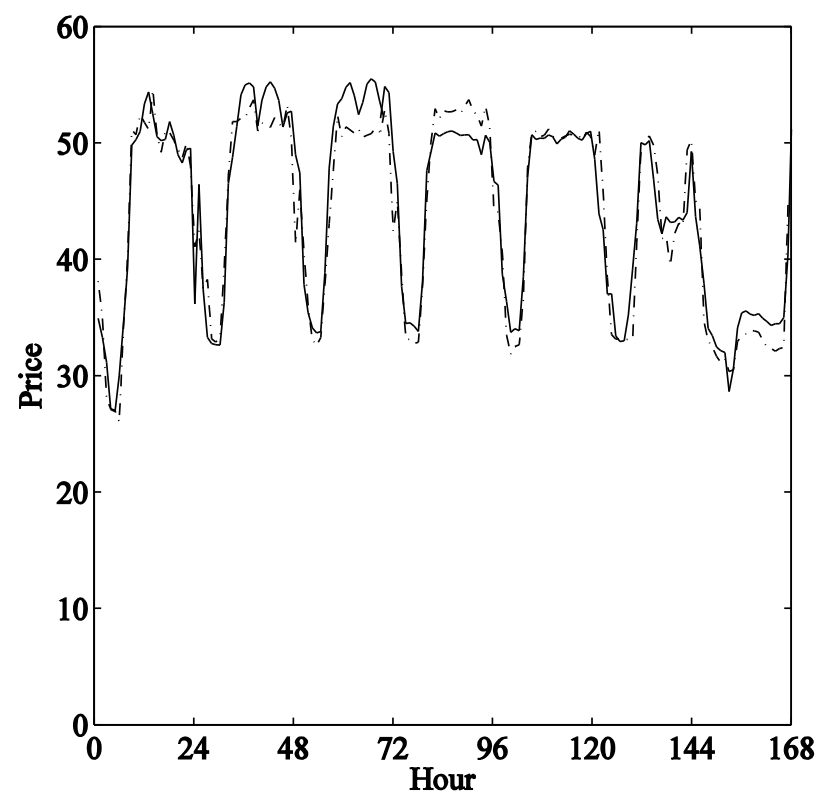

Fig. 5. Spring week: actual prices, solid line, together with the forecasted prices, dash-dot line, in euro per megawatt hour.

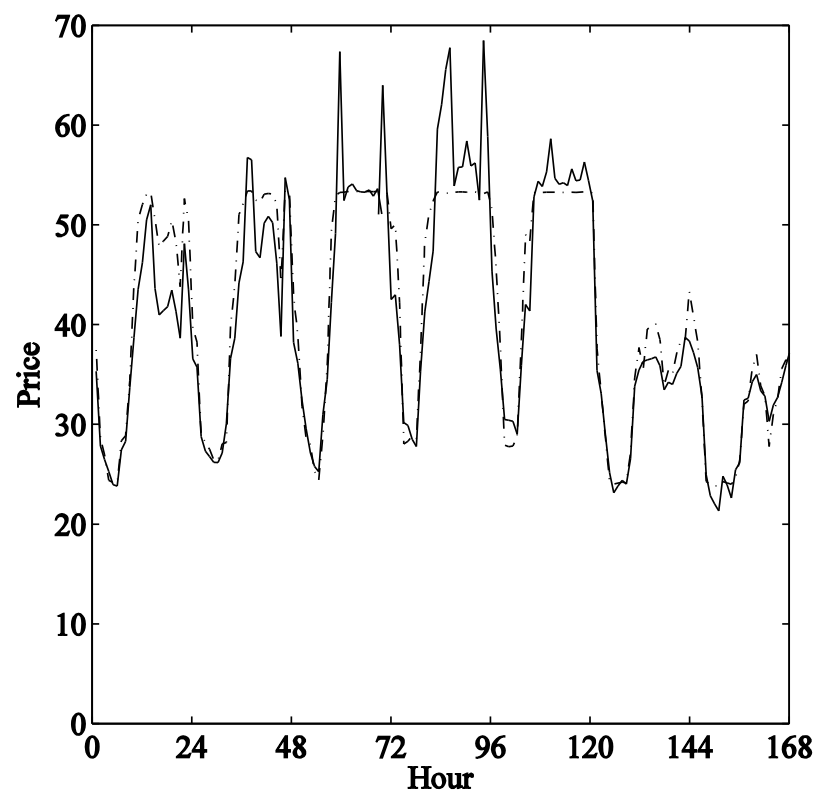

Fig. 6. Summer week: actual prices, solid line, together with the forecasted prices, dash-dot line, in euro per megawatt hour. 


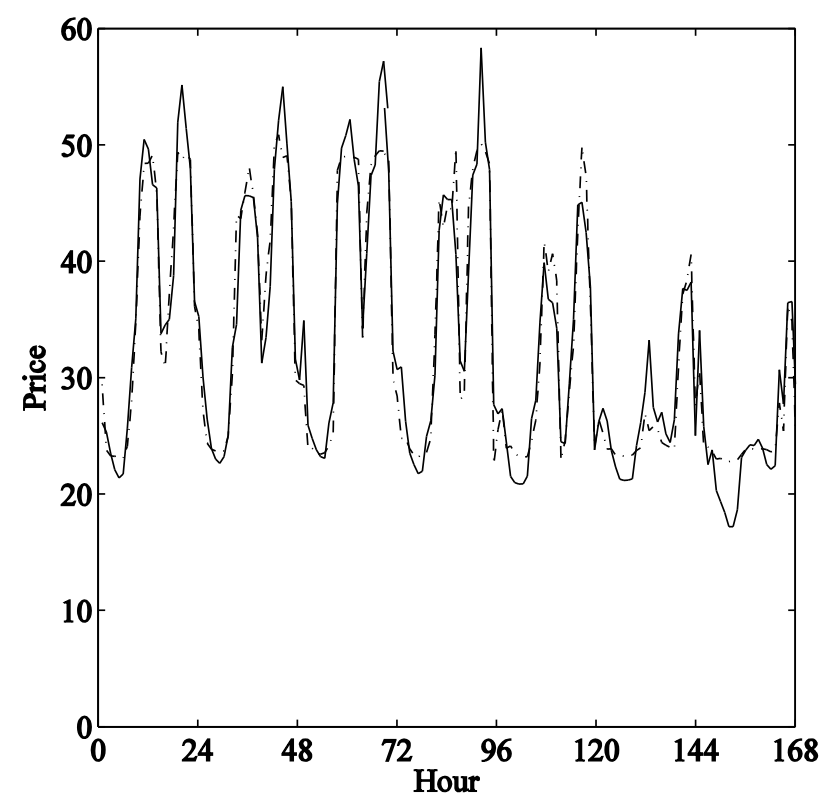

Fig. 7. Fall week: actual prices, solid line, together with the forecasted prices, dash-dot line, in euro per megawatt hour.

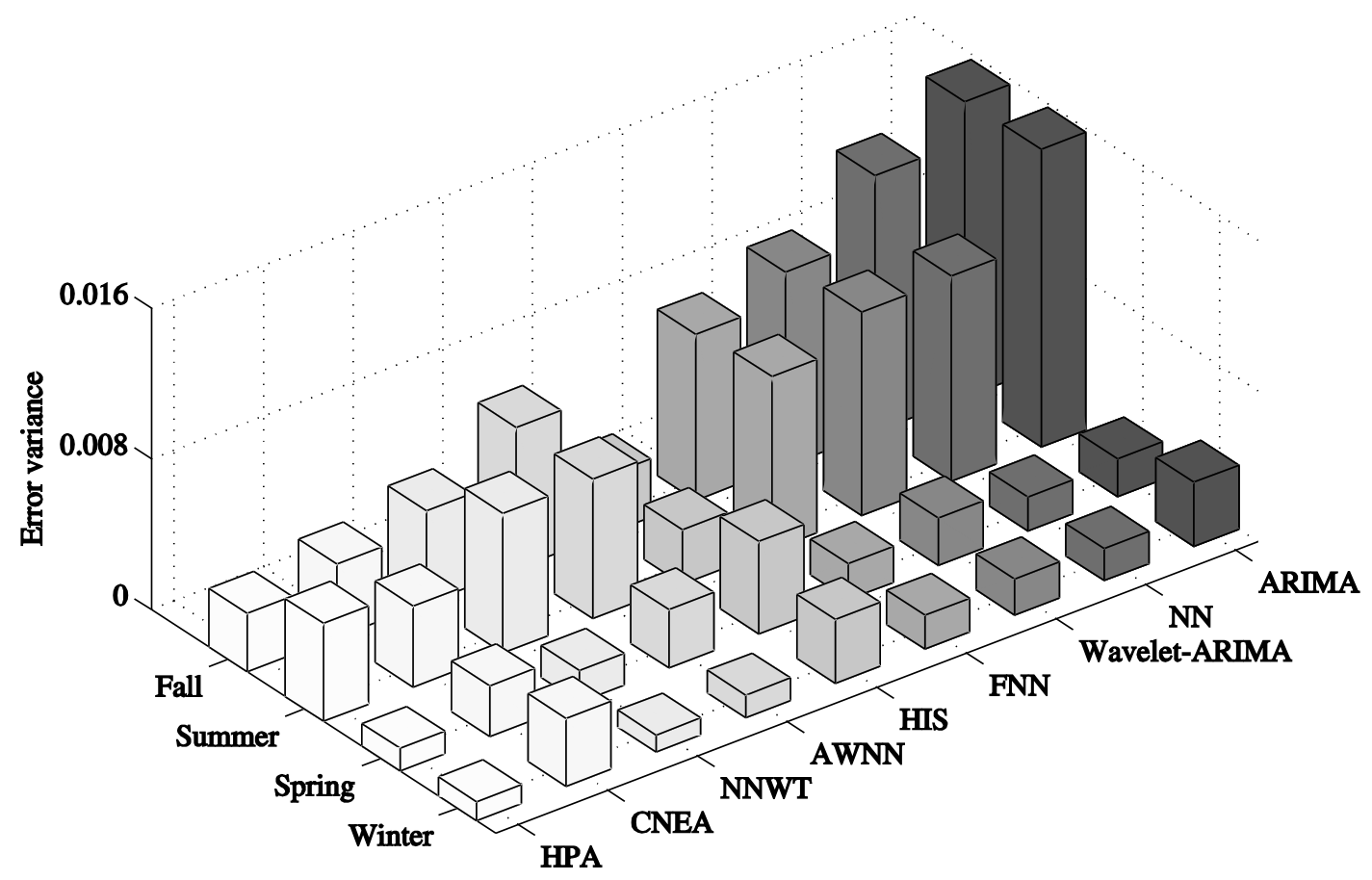

Fig. 8. Comparative error variance results. 


\section{Tables}

Table 1

Parameters of the PSO algorithm

\begin{tabular}{c|c}
\hline Parameters & Value \\
\hline Number of particles & 25 \\
\hline Number of iterations & 2000 \\
\hline Cognitive acceleration $C_{1}$ & 2.0 \\
\hline Social acceleration $C_{2}$ & 2.0 \\
\hline Initial inertia weight $\omega_{\min }$ & 0.9 \\
\hline Final inertia weight $\omega_{\max }$ & 0.4 \\
\hline
\end{tabular}

Table 2

Statistical analys is of the weekly forecasting error

\begin{tabular}{cccc}
\hline Week & MAPE $(\%)$ & $\sqrt{\text { SSE }}$ & SDE \\
\hline Winter & 3.65 & 27.39 & 1.38 \\
Spring & 4.19 & 31.38 & 1.54 \\
Summer & 6.76 & 51.95 & 2.93 \\
Fall & 6.53 & 36.86 & 1.85 \\
\hline
\end{tabular}

Table 3

Comparative MAPE (\%) results

\begin{tabular}{cccccc}
\hline & Winter & Spring & Summer & Fall & Average \\
\hline ARIMA [13] & 6.32 & 6.36 & 13.39 & 13.78 & 9.96 \\
Mixed-model [15] & 6.15 & 4.46 & 14.90 & 11.68 & 9.30 \\
NN [16] & 5.23 & 5.36 & 11.40 & 13.65 & 8.91 \\
Wavelet-ARIMA [14] & 4.78 & 5.69 & 10.70 & 11.27 & 8.11 \\
WNN [19] & 5.15 & 4.34 & 10.89 & 11.83 & 8.05 \\
FNN [18] & 4.62 & 5.30 & 9.84 & 10.32 & 7.52 \\
HIS [21] & 6.06 & 7.07 & 7.47 & 7.30 & 6.97 \\
AWNN [20] & 3.43 & 4.67 & 9.64 & 9.29 & 6.75 \\
NNWT [17] & 3.61 & 4.22 & 9.50 & 9.28 & 6.65 \\
CNEA [22] & 4.88 & 4.65 & 5.79 & 5.96 & 5.32 \\
HPA & 3.65 & 4.19 & 6.76 & 6.53 & 5.28 \\
\hline
\end{tabular}


Table 4

Average weekly error variance

\begin{tabular}{cc}
\hline & Average \\
\hline ARIMA [13] & 0.0092 \\
NN [16] & 0.0070 \\
Wavelet-ARIMA [14] & 0.0064 \\
FNN [18] & 0.0054 \\
HIS [21] & 0.0036 \\
AWNN [20] & 0.0048 \\
NNWT [17] & 0.0037 \\
CNEA [22] & 0.0036 \\
HPA & 0.0026 \\
\hline
\end{tabular}

